\title{
Evaluation of a simulation-based curriculum for implementing a new clinical protocolis
}

\author{
David Marzano a,*, Roger Smith a , Jill M. Mhyre ${ }^{\text {b }}$, F. Jacob Seagull a , Diana Curran ${ }^{\text {a }}$, Sydney Behrmann ${ }^{\text {a }}$, \\ Kristina Priessnitz ${ }^{a}$, Maya Hammoud ${ }^{a}$ \\ a University of Michigan Medical School, Ann Arbor, Michigan, USA \\ b University of Arkansas for Medical Sciences, Little Rock, Arkansas, USA
}

\section{A R T I C L E I N F O}

\section{Article history:}

Received 15 March 2016

Received in revised form 18 May 2016

Accepted 22 August 2016

\section{Keywords:}

Cesarean delivery urgency classification

Obstetrics

Protocol implementation

Simulation

\begin{abstract}
A B S T R A C T
Objective: To evaluate the implementation of a new clinical protocol utilizing on-unit simulation for team training. Methods: A prospective observational study was performed at the obstetrics unit of Von Voightlander Women's Hospital, Michigan, USA, between October 1, 2012 to April 30, 2013. All members of the labor and delivery team were eligible for participation. Traditional education methods and in-situ multi-disciplinary simulations were used to educate labor and delivery staff. Following each simulation, participants responded to a survey regarding their experience. To evaluate the effect of the interventions, paging content was analyzed for mandated elements and adherence to operating room entry-time tracking was examined. Results: In total, 51 unique individuals participated in 12 simulations during a 6-month period. Simulation was perceived as a valuable activity and paging content improved. Following the intervention, the inclusion of a goal time for reaching the operation room increased from $7 \%$ to $61 \%$ of pages and the proportion of patients entering to operating room within 10 minutes of the stated goal increased from $67 \%$ to $85 \%$. Conclusion: The training program was well received, and the accuracy of the communication and the goal set for reaching the operating room improved.

(C) 2016 International Federation of Gynecology and Obstetrics. Published by Elsevier Ireland Ltd. All rights reserved.
\end{abstract}

\section{Introduction}

The Joint Commission, USA, has identified failures in communication as the root cause of nearly $50 \%$ of maternal adverse events and more than $65 \%$ of perinatal deaths and injuries between 2004 and 2013 [1]. Both the American College of Obstetricians and Gynecologists and the Joint Commission now recommend simulation and team training to improve team communication and performance [2]. Simulation-based team training is a valuable platform for developing, maintaining, and evaluating both individual clinical skills and team performance [3]. It also can identify discrepancies in policy between institutional and departmental practices, and is useful in correcting latent hazards that increase risks for system failures and patient harm [4]. In comparison with traditional didactic approaches, simulation promotes active learning and includes opportunities for hands-on practice [5].

Coordination of cesarean delivery is a complex task for obstetric teams. Effective communication, teamwork, and appropriate timing

\footnotetext{
is Presented at ACOG 2015 Annual Clinical and Scientific Meeting; May 2-6, 2015; San Francisco, CA, USA (Abstract 25).

* Corresponding author at: Department of Obstetrics and Gynecology, University of Michigan Medical School, 1500 E. Medical Center Dr., L4000 Von Voigtlander Women's Hospital, Ann Arbor, MI, 48109, USA. Tel.: +1 734936 3110; fax: +1 7349367722.

E-mail address: damarz@med.umich.edu (D. Marzano).
}

are critical to the coordination of care. Standardized communication processes can help optimize care for patients needing surgery, and also for other patients on the unit requiring surgical intervention. Effective communication improves situational awareness. While a 30-minute decision-to-incision rule has been used traditionally as a standard for obstetric services [6], not all procedures are optimally performed within this time frame. According to the American College of Obstetricians and Gynecologists, the decision-to-incision interval and mode of delivery should be based on the timing that best incorporates maternal and fetal risks and benefits [7]. Traditionally, the obstetrician has decided upon the timing of surgery and the consultation of team members, including nursing and anesthesia, has been an afterthought. Whereas patient needs come first, consultation with anesthesia and nursing is critical to optimize the timing of surgery. In 2012, a multi-disciplinary leadership team at the University of Michigan Von Voightlander Women's Hospital revised institutional protocols to classify the level of surgical urgency and to coordinate unplanned surgical procedures.

Policy changes in a large organization are often not implemented effectively to result in true behavioral changes. This difficulty leads to the failure of policies and potentially detrimental outcomes for patients. The primary purpose of the present study was to evaluate the effectiveness of simulation as a method of introducing policy change. The secondary outcome was to assess if simulation, utilizing contextually accurate team training, would meet learner expectations in terms of being an effective means to learn and to comply with the new protocol. 


\section{Materials and methods}

The present prospective observational study was conducted between October 1, 2012 and April 30, 2013 at the obstetrics unit of the Von Voightlander Women's Hospital, Michigan USA, to study the use of simulation in the adoption of a new classification system based upon urgency for obstetric procedures. All members of the labor and delivery team were eligible for participation. This included faculty, residents, fellows, and medical students from the departments of obstetrics and anesthesia. All labor and delivery nurses and surgical technicians were considered eligible. The study was reviewed by the University of Michigan institutional review board and granted a status of not regulated because it was part of a quality improvement initiative; therefore, individual consent was not required.

The study center, Von Voightlander Women's Hospital, consists of a 50-bed unit (30 labor, delivery, recovery, and postpartum beds, seven prepartum beds, 13 traditional postpartum beds), and four operating rooms. One fully-equipped labor room was designated as an in-situ simulation center.

Historically, the study center used the "Birth Center Pager" (BCP) as an emergency pager system. The BCP was used for emergent cesarean deliveries only and provided a means of notifying all team members of patients proceeding directly to the operating room. After several adverse events involving patients requiring surgery urgently being delayed for an inappropriate amount of time, the system was revised to utilize the BCP for all unscheduled cesarean deliveries. Descriptive language was used to classify timing of procedures, leading to the use of, "emergent" and "urgent, non-emergent" categories. Any team member could activate the BCP and the following information was supposed to be included: urgency, patient name, planned procedure, and surgical goal time. Compliance with the inclusion of necessary paging elements was sporadic and goal entry time was interpreted variably, leading to confusion.

In 2012, owing to this lack of standardization, a new classification system was adopted for describing procedural urgency that included a modified version of the Royal College of Obstetricians and Gynaecologists' four-tiered urgency-classification system [8,9]; this classification system defines four urgency categories: (1) immediate threat to life of woman or fetus; (2) maternal or fetal compromise that is not immediately life-threatening; (3) needing early delivery but no maternal or fetal compromise; and (4) at a time to suit the woman and maternity team. This validated classification system was applied with two modifications. First, to avoid confusion with the National Institute of Child Health and Human Development fetal heart rate categories [10], the scale was modified to replace numeric categories with descriptive terms, "emergent", "urgent", "add-on", and "elective" (Table 1). Second, to apply the urgency scale to all surgical procedures, the term "delivery" was replaced with "surgery". The new operating room procedure communication protocol (Box 1) prescribed using the BCP to alert all team members simultaneously for all urgency levels except for scheduled surgeries. For emergent procedures, activation of the BCP alerts team members to proceed directly to the operating room. For urgent and add-on procedures, the protocol prescribes that team members decide on the urgency, and choose an appropriate time of entry into the operating room for each patient, with the goal of achieving entry time as close to the stated goal as possible. The protocol also mandates what information is paged to the team.

Table 1

New surgical classification.

\begin{tabular}{ll}
\hline Class & Definition \\
\hline Emergent & Immediate threat to life (well-being) of mother or baby \\
Urgent & Maternal or fetal compromise that is not immediately life threatening \\
Add-on & Clinical condition requiring surgery within a reasonable amount of time \\
Elective & Scheduled surgery, delay of surgery is considered safe for mother and baby \\
\hline
\end{tabular}

\section{Box 1}

Scripted communication protocols and procedures. Abbreviation: OR, operating room.

\section{Emergent}

- Procedure will begin as soon as possible.

- Birth Center Pager activation:

- "EMERGENT Room 50 SimMom, bradycardia moving to OR"

- Team meets in the emergency OR flagged by scrub technician.

Urgent

- Obstetrician:

${ }^{\circ}$ Calls anesthesiologist to discuss medical concerns; create situational awareness; decide on time of entry into the OR.

- Birth Center Pager activation:

- "URGENT Rm 50, SimMom, C/S for arrest of descent. Enter OR at 3:10pM. Dr. Smith, 555-5555."

- Anesthesia: communicate to scrub technician.

- Scrub technician: flags OR venue; team uses visual cue to meet in designated OR.

- Bedside nurse:

${ }^{\circ}$ Notifies charge nurse.

- Notifies pediatric or neonatal intensive care unit team for delivery upon entry into the OR.

Add-on

- Obstetrician:

o Calls anesthesiologist to discuss medical concerns; creates situational awareness; decide on time of entry into the OR.

- Birth Center Pager activation:

- "ADD-ON Rm 50, SimMom, Prior C/S, PPROM, not in

labor. Enter OR at 3:10PM. Dr. Smith, 555-5555."

- Anesthesia: communicates to scrub technician.

- Scrub technician: Flags OR venue; team uses visual cue to meet in designated OR

- Beside nurse:

o Notifies charge nurse.

o Notifies pediatric or neonatal intensive care unit team for delivery upon entry into the OR.

Elective

- OR team leader will monitor resources and team availability.

- Group pager NOT utilized.

- Team leader calls individuals for meeting time; confirms availability.

- Team meets at designated time in OR for pre-anesthetic time out.

A multi-pronged approach was used to implement the new process. Traditional instruction was delivered via didactic presentations at morbidity and mortality conferences, nursing conferences, reviews at team sign-outs, and by email. Placards with the protocol were also posted in high traffic areas. These more-traditional methods of implementation took place over a 2-month period, during which, four pilot simulation sessions were delivered. Feedback from department-wide presentations and from these pilot simulations was used to optimize the training sessions.

The simulation sessions employed a high-fidelity obstetrics simulator (SimMom; Laerdal, Wappingers Falls, New York, USA) and sessions were conducted twice monthly, for 6 months. Outlines of the 12 simulation sessions (each consisting of two scenarios) are included in Table 2. The study researchers led each session and participation by learners, representing all members of the care delivery team, was solicited. Obstetrics faculty and residents were scheduled to be included in sessions. Nurses participated while on duty, when staffing levels permitted. Participation by anesthesiologists, surgical technicians, and clerks was voluntary and solicited when they were on duty. 
Table 2

Simulation training procedures.

\begin{tabular}{|c|c|c|}
\hline \multirow[t]{2}{*}{ Session } & \multicolumn{2}{|l|}{ Simulated scenario } \\
\hline & First simulation & Second simulation \\
\hline \multicolumn{3}{|c|}{ Announced drills } \\
\hline 1 & Arrest of descent (add-on) & Patient request (elective) \\
\hline 2 & Cord prolapse (emergent) & Abruptio placentae (emergent) \\
\hline 3 & $\begin{array}{l}\text { Repeat cesarean delivery ruptured } \\
\text { not in labor (add-on) }\end{array}$ & Uterine rupture (emergent) \\
\hline 4 & $\begin{array}{l}\text { Repeat cesarean delivery ruptured in } \\
\text { labor (urgent) }\end{array}$ & $\begin{array}{l}\text { National Institute of Child Health and } \\
\text { Human Development category II } \\
\text { tracing (urgent) }\end{array}$ \\
\hline 5 & $\begin{array}{l}\text { Repeat cesarean delivery not } \\
\text { ruptured in labor (urgent) }\end{array}$ & Fetal bradycardia (emergent) \\
\hline 6 & Breech in active labor (urgent) & Eclamptic seizure (emergent) \\
\hline \multicolumn{3}{|c|}{ Unannounced drills } \\
\hline 7 & Eclamptic seizure (emergent) & Breech in active labor (urgent) \\
\hline 8 & Fetal bradycardia (emergent) & $\begin{array}{l}\text { Repeat cesarean delivery not } \\
\text { ruptured in labor (urgent) }\end{array}$ \\
\hline 9 & $\begin{array}{l}\text { National Institute of Child Health and } \\
\text { Human Development category II } \\
\text { tracing (urgent) }\end{array}$ & $\begin{array}{l}\text { Repeat cesarean delivery ruptured in } \\
\text { labor (urgent) }\end{array}$ \\
\hline 10 & Uterine rupture (emergent) & $\begin{array}{l}\text { Repeat cesarean delivery ruptured } \\
\text { not in labor (add-on) }\end{array}$ \\
\hline 11 & Abruptio placentae (emergent) & Cord prolapse (emergent) \\
\hline 12 & Patient request (elective) & Arrest of descent (add-on) \\
\hline
\end{tabular}

Preceding each simulation, participants completed an on-line educational module reviewing the new classification system, basic team dynamics, and communication techniques based upon TeamSTEPPS protocols [11]. Each session was 2 hours in length including a briefing, a didactic review and a discussion of the on-line educational material, two simulation scenarios, and a debriefing.

Following each simulation, participants completed a survey of their experience regarding the value of the simulation and the new communication protocol using a five-point Likert scale. Changes in behavior were assessed by analyzing actual paging content before and after the training for urgent and emergent procedures. The elements tracked included: (1) urgency indication; (2) patient identification; (3) surgery indication; (4) goal entry time (this was measured only for urgent-level pages because the expectation was that emergent-level pages would move to the operating room immediately); and (5) activator contact information. Additionally, a comparison was made of the target operatingroom entry time and the actual operating-room entry time, recorded in the anesthesia record for urgent procedures. Statistical analyses were performed with SAS version 9.3 (SAS Institute, Cary, NC, USA).

\section{Results}

A total of 51 unique individuals participated in the 12 simulation sessions, with several individuals attending multiple training sessions. The representation of different disciplines at the training sessions is outlined in Table 3.

Table 3

Attendance and representation at simulation sessions of different disciplines.

\begin{tabular}{lll}
\hline Discipline & $\begin{array}{l}\text { No. of unique individuals who attended } \\
(\mathrm{n}=51)\end{array}$ & Representation, \% \\
\hline Obstetrics faculty & 13 & 100 \\
Obstetric residents & 12 & 100 \\
Anesthesiology faculty & 2 & 42 \\
Anesthesiology residents & 9 & 75 \\
Nursing staff & 13 & 83 \\
Surgical technicians & 1 & 8 \\
Medical students & 1 & 8 \\
\hline
\end{tabular}

a Representation was calculated by dividing the number of sessions attended by at least one member of a discipline by total number of simulation sessions held (12).
Table 4

Perceptions of simulation training $(n=51)$

\begin{tabular}{ll}
\hline Simulation activity statement & Rating $^{\mathrm{ab}}$ \\
\hline Simulation helped in learning the new communication protocol & 3.9 \\
Simulation helped the team work together & 3.8 \\
Simulation training Improved the ability to coordinate the team & 3.8 \\
Simulation has helped effectively implement the new communication & 3.6 \\
$\quad$ process & 3.6 \\
Simulation activities (in-situ) won't cause harm by distracting the clinical & \\
$\quad$ team & 4.0 \\
Simulation was a good use of time & 3.9 \\
\hline I could focus on the simulation despite clinical responsibilities &
\end{tabular}

a Ratings were provided using a five-point Likert scale with higher numbers indicating stronger agreement.

b Values are given as means.

Simulation was perceived as a valuable activity, as demonstrated by the mean Likert-scale responses shown in Table 4. Participants reported that they felt simulation helped them learn the new communication process (3.9), helped teamwork (3.8), and helped team coordination (3.8). Participants agreed that the simulation made a difference in implementing the new protocol (3.6). Additionally, the simulation was perceived as not causing a distraction and interfering with patient care (3.6), and it was considered a good use of time (4.0).

Perceptions of the new protocol were positive (Table 5). Participants reported the new communication process was helpful in coordinating operating room teams (4.0) and could help to prevent delays (3.8). Similarly, learners felt that the new process was not too complicated (reverse coded) (2.4) and that the standard communication did not work well without the new process (reverse coded) (2.3).

The comparison of paging content from 3 months before the implementation of the program with 6 months afterwards demonstrated improvement in the inclusion of the necessary specified elements. For urgent pages, there was an overall increase in the percentage of pages that included patient identification (32/42 [76\%] vs 48/54 [89\%]), surgical indication (40/42 [95\%] vs 53/54 [98\%]), and goal operating room entry time (3/42 [7\%] vs 33/54 [61\%]). For emergent pages, the percentage of pages with the urgency level stated increased from 9 of $15(60 \%)$ to 18 of $28(64 \%)$ and the percentage including surgical indication increased from 13 of 15 (87\%) to 27 of 28 (96\%); the goal operating room entry time and activator contact information were not required in the new protocol and so were not analyzed. Among emergent pages, a decrease in the percentage of pages with patient identification (7/15 [47\%] vs 9/28 [32\%]) was observed.

The examination of actual operating-room entry times for urgent surgeries demonstrated that the percentage of pages resulting in operating room entry within 10 minutes of the stated goal increased from 20 of $30(67 \%)$ to 28 of 33 (85\%), a noticeable improvement in the percentage of procedures adhering to the stated goal entry time.

Debriefing sessions uncovered interesting deficiencies that were anticipated at the present study at the outset. The two most frequent issues identified during debriefing were that some team members were unfamiliar with how to activate the $\mathrm{BCP}$, and that there was confusion regarding the operating room that would be used for patients following paging.

Table 5

Perceptions of new communication system $(n=51)$.

\begin{tabular}{ll}
\hline Communication protocol statement & Rating $^{\mathrm{ab}}$ \\
\hline The new communication process helps to coordinate operating-room teams & 4.0 \\
The new communication process can prevent delays & 3.8 \\
The new communication process is too complicated (reverse coded) & 2.4 \\
Standard communication works well enough without this new process & 2.3 \\
(reverse coded) & \\
\hline a Ratings were provided using a five-point Likert scale with higher numbers indicating \\
stronger agreement.
\end{tabular}




\section{Discussion}

The present study demonstrated that the implemented training program was well received and was effective in improving paging content; the identified paging elements were present in an increased percentage of pages after the intervention.

Successful implementation of new protocols is difficult; traditional passive learning methods have limited effectiveness for prompting individuals to adopt a new workflow [12]. As part of a major educational component aimed at implementing a new process for communicating the urgency of surgeries in the delivery center, the present study set out to examine the reception and effectiveness of a simulation-based curriculum. It was hypothesized that simulation training would be well received by participants as a tool for introducing and embedding a new classification paradigm into every-day practice.

The survey element of the present study demonstrated a high level of acceptance for the curriculum. Participants agreed that the simulations aided learners in understanding the new classification protocol and that the simulation training was a good use of time. These findings are meaningful; the effectiveness of team training programs has been shown to correlate with positive attitudes toward training [13]. The positive feedback elicited suggests that future policy and protocol changes would benefit from incorporating simulation into their implementation. This is consistent with previous findings demonstrating that didactic sessions are effective at increasing knowledge whereas simulation training has been shown to improve performance [14].

Participants agreed that simulation exercises enhanced collaboration among team members. A more favorable perception of team competency leads to greater confidence in a team, which can in turn lead to improved clinical outcomes being brought about by that team $[5,15]$. In the present study, where the healthcare team was comprised of varied professionals, the ability to manage team dynamics in delivering patient care is critical. Simulation creates a controlled environment to practice new skills, free of patients and family members [16]. This inherent benefit of simulation training likely influenced feelings of sureness and confidence in the learning process.

The analysis of paging elements demonstrated an increase in the inclusion of specified necessary paging elements following training. It is likely that this was due, at least in part, to the simulation-training program. This has important implications to communication throughout the care spectrum, including improving the shared mental model and situational awareness.

During the present study, the timeliness of entry to the operating room improved. This was congruent with the changes in the new protocol. The aim was to improve communication and situational awareness, beginning each surgical procedure in the appropriate amount of time, taking into account the acuity of all patients on the unit. This is in contrast to the rigid 30-minute decision-to-incision idea, and the authors suggest that the 30-minute approach should be abandoned.

Drilling clinical behaviors in-situ is important for translating skill training to patient care, and the on-site context of drills can also elucidate operational inefficiencies affecting the patient-care unit itself [16]. The present platform uncovered deficiencies that would not have surfaced when utilizing the traditional model for introducing new policies. Although not a goal of the present study, the debriefing identified that some team members were unfamiliar with how to activate the $\mathrm{BCP}$. Without the requirement of actually activating the BCP during a simulation, this deficiency would not have been identified. Additionally, through simulation, the challenge of communicating the selection of the appropriate operating room for an unscheduled urgent or emergent cesarean delivery was demonstrated. This experience helped make the placement of flags outside the operating room indicating where a patient should be rushed part of routine practice.

The authors acknowledge several limitations of the present study. Whereas the number of paging elements included in pages improved over the course of the training, this could be a result of increased awareness, and not the simulation itself. There was an unexpected decrease in patient identification included in emergency pages following the simulation training. An explanation for this finding is that clerical staff were frequently required to activate the BCP, providing further support for the necessity of including all members of the care team in training. Pages before implementation of the simulation training and during the subsequent 6 months were analyzed. A follow-up study is required to ensure that the improved behaviors have been sustained.

The importance of participation is critical; having representatives of all members of the team present for the simulations is essential. Obstetric faculty and house officers were present at every simulation session; however, the remainder of team was not as well represented. The lack of complete representation at simulations by nursing and anesthesiology was a limitation. Although in-situ simulation created the opportunity for some nursing and other labor and delivery staff to take part in training, protected time was not set aside for them to participate; lower participation rates were observed among these groups. Sustaining simulation programs with full team participation requires the institutional commitment of resources.

The simulation training was only conducted in one institution. The size and resources at a particular institution could influence the ability to provide adequate and successful simulation training; consequently, the translation of results of the present study to other institutions could be inappropriate. Finally, the scope of the present study only extended to the point of how the simulations were perceived and whether or not certain desired behaviors were applied; it was not designed to demonstrate whether simulations resulted in improved outcomes.

Despite these limitations, it is concluded that simulation is an effective tool to help implement new protocols that require complex, coordinated multi-disciplinary teamwork. As part of an on-going quality improvement cycle, changes have been made to the present training program. These have included involving clerical staff during training, increasing the participation of nursing staff, and ensuring simulations are a routine event at the study unit. Paging elements will continue to be monitored for sustainability. In the future, this tool could be critically important to the successful and safe implementation of new clinical pathways, new processes, and new electronic health records.

\section{Acknowledgements}

The present study was funded by a University of Michigan Clinical Simulation Center Grant.

\section{Conflict of interest}

The authors have no conflicts of interest.

\section{References}

[1] The Joint Commission. Sentinel Event Data. Root Causes by Event Type 2004-June 2013. http://www.medleague.com/wp-content/uploads/2013/11/Root_Causes_by_ Event_Type_2004-202013.pdf. Published 2013. Accessed August 22, 2016.

[2] American College of Obstetricians and Gynecologists Committee on Patient Safety and Quality Improvement. ACOG Committee Opinion No. 487: Preparing for clinical emergencies in obstetrics and gynecology. Obstet Gynecol 2011;117(4):1032-4.

[3] Andreatta P, Marzano D. Healthcare management strategies: interdisciplinary team factors. Curr Opin Obstet Gynecol 2012;24(6):445-52.

[4] Andreatta P, Frankel J, Boblick Smith S, Bullough A, Marzano D. Interdisciplinary team training identifies discrepancies in institutional policies and practices. Am J Obstet Gynecol 2011;205(4):298-301.

[5] Gum L, Greenhill J, Dix K. Clinical simulation in maternity (CSiM): interprofessional learning through simulation team training. Qual Saf Health Care 2010;19(5), e19.

[6] American College of Obstetricians and Gynecologists. Standards for obstetricgynecologic services. Sixth edition. Washington, DC: American College of Obstetricians and Gynecologists; 1985.

American Congress of Obstetricians and Gynecologists. Standards for Obstetrics and Gynecologic Services. Sixth ed. Washington DC: The College; 1988.

[7] American College of Obstetricians and Gynecologists. Practice bulletin no. 116: Management of intrapartum fetal heart rate tracings. Obstet Gynecol 2010;116(5): 1232-40. 
[8] Lucas DN, Yentis SM, Kinsella SM, Holdcroft A, May AE, Wee M, et al. Urgency of caesarean section: a new classification. J R Soc Med 2000;93(7):346-50.

[9] Brennand JE, Millns P, Yentis S, Hinshaw HK. Classification of Urgency of Caesarean Section - a Continuum of Risk (Good Practice No. 11). London: Royal College of Obstetricians and Gynaecologists; 2010.

[10] American College of Obstetricians and Gynecologists. ACOG Practice Bulletin No. 106: Intrapartum fetal heart rate monitoring: nomenclature, interpretation, and general management principles. Obstet Gynecol 2009;114(1):192-202.

[11] Agency for Healthcare Research and Quality. TeamSTEPPS Essentials Instructional Module and Course Slides. http://www.ahrq.gov/professionals/education/curriculumtools/teamstepps/instructor/essentials/index.html. Published 2014. Accessed August 22, 2016.

[12] Mclvor W, Burden A, Weinger MB, Steadman R. Simulation for maintenance of certification in anesthesiology: the first two years. J Contin Educ Health Prof 2012; 32(4):236-42.
[13] Sevdalis N, Hull L, Birnbach DJ. Improving patient safety in the operating theatre and perioperative care: obstacles, interventions, and priorities for accelerating progress. Br J Anaesth 2012;109(Suppl 1):i3-i16.

[14] Daniels K, Arafeh J, Clark A, Waller S, Druzin M, Chueh J. Prospective randomized trial of simulation versus didactic teaching for obstetrical emergencies. Simul Healthc 2010;5(1):40-5.

[15] Sexton JB, Helmreich RL, Neilands TB, Rowan K, Vella K, Boyden J, et al. The Safety Attitudes Questionnaire: psychometric properties, benchmarking data, and emerging research. BMC Health Serv Res 2006;6:44.

[16] Argani CH, Eichelberger M, Deering S, Satin AJ. The case for simulation as part of a comprehensive patient safety program. Am J Obstet Gynecol 2012;206(6):451-5. 